\title{
UTILIDAD DE LA EXPRESIÓN DE Ki67 EN LAS BIOPSIAS PREOPERATORIAS PARA PREDECIR LA RECIDIVA BIOQUÍMICA DEL CÁNCER DE PRÓSTATA DESPUÉS DE LA PROSTATECTOMÍA RADICAL
}

\author{
A. OJEA CALVO, M.J. MOSTEIRO CERVIÑO, F. DOMÍNGUEZ FREIRE, \\ A. ALONSO RODRIGO, B. RODRÍGUEZ IGLESIAS, J. BENAVENTE DELGADO, \\ J.M. BARROS RODRÍGUEZ, A. GONZÁLEZ PIÑEIRO
}

Servicio de Urología. Hospital Universitario Xeral-Cíes. Vigo (Pontevedra).

Actas Urol Esp. 28 (9): 650-660, 2004

\section{RESUMEN}

UTILIDAD DE LA EXPRESIÓN DE Ki67 EN LAS BIOPSIAS PREOPERATORIAS PARA PREDECIR LA RECIDIVA BIOQUÍMICA DEL CÁNCER DE PRÓSTATA DESPUÉS DE LA PROSTATECTOMÍA RADICAL

OBJETIVO: Evaluar la utilidad de la expresión de Ki67 de las biopsias diagnósticas preoperatorias, para predecir la recidiva bioquímica del cáncer de próstata después de la prostatectomía radical.

MATERIAL Y MÉTODOS: Analizamos la expresión de Ki67 en las biopsias ecodirigidas de 103 pacientes a los que se les practicó prostatectomía radical. El tiempo medio de seguimiento es de 3,4 años (1,3-8,8 años). Correlacionamos la recidiva bioquímica con los factores pronósticos clásicos como el PSA $(>10 / \leq 10)$, Gleason $(\geq 7 /<7)$, clasificación pT (pT3/pT0-2) y el factor pronóstico inmunohistoquímico Ki67 (>3\%/ $\leq 3 \%)$.

RESULTADOS: De los 103 pacientes, 71 (69\%) no recidivaron y en 32 (31\%) se detectó recidiva bioquímica. El PSA medio preoperatorio era de $10,7 \mathrm{ng} / \mathrm{ml}$ en los pacientes sin recidiva y de $20,90 \mathrm{ng} / \mathrm{ml}$ en los pacientes con recidiva bioquímica $(p=0,0001)$. La media de la suma del Gleason era de 6,03 en los pacientes sin recidiva y de 6,75 en los pacientes con recidiva bioquímica ( $\mathrm{p}=0,0001)$. El porcentaje de expresión de Ki67 era de 3,95\% en los pacientes sin recidiva frente al 5,05 de los pacientes con recidiva bioquímica. Los tumores pT0-2 progresaron $12 / 67(17,9 \%)$ y los tumores pT3 20/36 (55,6\%) (p=0,0001). $\mathrm{El}$ análisis multivariante indica que no existe una relación entre el Ki67 (>3\%/ $\leq 3 \%)$ del material de las biopsias preoperatorias y la recidiva bioquímica del cáncer de próstata después de la prostatectomía radical $(\mathrm{p}=0,204)$.

CONCLUSIONES: El factor pronóstico inmunohistoquímico Ki67 ( $>3 \% / \leq 3 \%)$ en la biopsia preoperatoria es menos eficaz que los factores clásicos, PSA $(>10 / \leq 10)$, Gleason $(\geq 7 /<7)$ y clasificación pT (pT3/pT0-2), para predecir la progresión bioquímica del cáncer de próstata después de la prostatectomía radical.

PALABRAS CLAVE: Cáncer de próstata. Prostatectomía radical. Recidiva bioquímica. Factores pronósticos. Ki67.

\begin{abstract}
THE USEFULNESS OF KI67 EXPRESSION IN THE BIOPSY SPECIMENS, TO PREDICT THE BIOCHEMICAL PROGRESION OF THE PROSTATE CANCER AFTER RADICAL PROSTATECTOMY

OBJETIVE: To evaluate the usefulness of Ki67 expression in the biopsy specimens, to predict the biochemical progression of the prostate cancer after radical prostatectomy.

MATERIAL AND METHODS: We analyse the Ki67 expression in the biopsy specimens of 103 patients treated with radical prostatectomy. The mean follow up is 3.4 years (1.3-8.8 years). We correlate the biochemical progression with traditional prognostic factors as the PSA $(>10 / \leq 10)$, Gleason $(\geq 7 /<7)$, pT classification (pT3/pT0-2) and immunohistochemical factor Ki67 $(>3 \% / \leq 3 \%)$.

RESULTS: Of all 103 patients, in 71 (69\%) biochemical progression was not detected and in 32 (31\%) biochemical progression was detected. The mean of preoperative PSA is $10.07 \mathrm{ng} / \mathrm{ml}$ in the patients without progression and $20.90 \mathrm{ng} / \mathrm{ml}$ in the patients with biochemical progression $(\mathrm{p}=0.0001)$. The mean of Gleason score in 6.03 in the patients without progression and 6.75 in the patients with biochemical progression ( $\mathrm{p}=0.0001$ ). The percentage of Ki67 expression is $3.95 \%$ in the patients without progression and $5.05 \%$ in the patients with biochemical progression $(\mathrm{p}=0.030)$. The tumors pT0-2 progressed $12 / 67(17.9 \%)$ and the tumors pT3 progressed 20/36 $(55.6 \%)(\mathrm{p}=0.0001)$. Multivariant regression analysis indicate that it does not exist a statistically significant relation between Ki67 $(>3 \% / \leq 3 \%)$ expression in the biopsy specimens and the biochemical progression of the prostate cancer after radical prostatectomy $(\mathrm{p}=0.204)$.

CONCLUSIONS: The immunohistochemical factor Ki67 $(>3 \% / \leq 3 \%)$ in the biopsy specimens, is less effective than the classic factors, PSA $(>10 / \leq 10)$, Gleason $(\geq 7 /<7)$ and pT classification $(\mathrm{pT} 3 / \mathrm{pTO}-2)$, to predict the biochemical progression of the prostate cancer after radical prostatectomy.

KEY WORDS: Prostate cancer. Radical prostatectomy. Biochemical progression. Prognostic factors. Ki67.
\end{abstract}


$\mathrm{L}$ as opciones terapéuticas con intención curativa del cáncer de próstata localizado, prostatectomía radical y radioterapia externa, no están exentas de efectos secundarios, de ahí la importancia de un correcto estadiaje preoperatorio, para evitar tratamientos inútiles y la consiguiente yatrogenia de los mismos. Con los factores pronósticos clásicos, entre el 29\% y el 59\% de los cánceres de próstata tratados con prostatectomía radical, por estar catalogados clínicamente como organoconfinados, sobrepasan la cápsula prostática en el estudio histopatológico de la pieza quirúrgica y la recidiva bioquímica del cáncer de próstata después de la prostatectomía radical se sitúa en torno al 30\% de los pacientes. Los factores pronósticos que habitualmente se vienen empleando se basan en criterios clínicos como la clasificación clínica, analíticos como el PSA y anatomopatológicos como la cantidad de tumor en la biopsia preoperatoria, el Gleason, el estadio anatomopatológico o la invasión perineural y vascular ${ }^{1-5}$.

Asociados a los factores pronósticos clásicos del cáncer comienzan a situarse nuevos factores pronósticos que están relacionados con la estructura celular, la dinámica de la célula y sus ciclos, con la muerte celular por apoptosis y con la interfase o relación de la célula con el estroma que la rodea. A la estructura celular están asociadas proteinas estructurales. La sobre-expresión de las proteínas Catepsina D, EGFR, Proteína $P$, cerb2, es indicativo de mal pronóstico y la sobreexpresión de $p \mathrm{~S} 2$ de buen pronóstico. En ciclo celular están involucradas las proteínas AgNORs, el antígeno nuclear proteico Ki67 y las Ciclinas que actúan a través de las Kinasas en todas las fases del ciclo celular. La sobre-expresión o porcentaje de células positivas sugieren una dinámica celular acelerada. A la apoptosis o muerte celular están asociadas las proteínas p27, p53 y BcL2. A la interfase célula estroma se asocian moléculas que intervienen en la neoangiogénesis y en las micrometástasis. Las moléculas de adhesión más importantes son la Cadherina E, antimetástasis y buen pronóstico; la Tenascina, que disminuye la permeabilidad del estroma y que es un dato de mal pronóstico; las metaloproteínas mm23 y sLEX son substancias proteinoliticas segregadas por el tumor, cuya sobreexpresión es un dato de mal pronóstico ${ }^{6-12,14-51}$.
El Ki67 es una proteína nuclear con un peso molecular que oscila entre 345 y 395 KDa. Es un antígeno nuclear que se expresa en las células en fase G1, S, G2, M y no se expresa en las células en fase G0, ya que se cataboliza rápidamente al final de la fase $\mathrm{M}$ y no se sintetiza en la fase G0. La expresión de Ki67 da una idea por lo tanto del estado proliferativo de la célula tumoral y su dinámica ${ }^{6-12,14-51}$.

El objetivo de este trabajo es evaluar la utilidad de la expresión de Ki67 en las biopsias diagnósticas preoperatorias, como factor pronóstico para predecir la recidiva bioquímica del cáncer de próstata después de la prostatectomía radical. ¿Puede suplantar el Ki67 a los factores pronósticos clásicos o enriquecerlos de tal modo que su determinación sirva para modificar actitudes terapéuticas?.

\section{MATERIAL Y MÉTODOS}

Pacientes. Se analizan 103 pacientes con cáncer de próstata en estadio clínico T1-2NOMO, diagnosticados por biopsia sextante ecodirigida, a los que se le había practicado prostatectomía radical retropúbica; $62(60 \%)$ pacientes se clasificaron preoperatoriamente como T1, 41(40\%) como T2. La edad media de los pacientes era de 62,39 años (rango 49-72, mediana 63.50, desv. tip. 6,071). Fueron intervenidos entre enero de 1995 y diciembre de 2002. La revisión se hizo en abril de 2004. El tiempo medio de seguimiento de los pacientes es de 3,4 años (rango 1,3-8,8; mediana 2,6 años; desv. tip. 1,9).

PSA preoperatorio. El PSA medio preoperatorio era de 13,44 (rango 1-71, mediana 9, desv. tip. 12,875). El PSA se determinó con el ensayo KRYPTOR $^{\mathrm{R}}$ que se fundamenta en la tecnología TRACE $^{\mathrm{R}}$ (Time Resolved Amplified Cryptate Emission).

Clasificación clínica y anatomopatológica. Los tumores se reclasificaron según el sistema TNM sexta edición de 2002 UICC $^{13}$. Los criterios minimos para la clasificación clínica fueron el tacto rectal, la ecografía transrectal y la linfadenectomía pélvica. Si el PSA era superior a $20 \mathrm{ng} / \mathrm{ml}$ se completaba el estudio preoperatorio con gammagrafía ósea y si el PSA era superior a $10 \mathrm{ng} / \mathrm{ml} \mathrm{se}$ 
hacía análisis peroperatorio de los ganglios. Si los ganglios en el estudio peroperatorio eran positivos se suspendía la intervención.

Las piezas de prostatectomía siempre se fijan durante 24 horas en formol tamponado al 10\%; se miden tres dimensiones, se pesan y se pintan todos los márgenes quirúrgicos con tinta china. Una vez seca, apoyando la pieza sobre su superficie posterior, se secciona dando cortes paralelos cada $4 \mathrm{~mm}$ en un plano trasversal, desde el ápex hasta la base. Las porciones cónicas resultantes del corte en la zona del ápex y base, se cortan de forma perpendicular al corte previo, para poder valorar más superficie quirúrgica de dichos bordes de resección. Se realiza inclusión parcial, con identificación individualizada de cada cápsula, con especial muestreo de: 1) cuello vesical, si está incluido; 2) ápex; 3) porciones posterolaterales de ambos lados, con muestreo de plexos vasculonerviosos; 4) zonas prostáticas sospechosas, tanto macroscópicamente como por la clínica o biopsias previas; 5) zonas de vesícula seminales o eyaculadores; 6) zona fibromuscular anterior, sobre todo en los casos de inicio de la neoplasia a la zona de transición.

Cada bloque de tejido procesado en parafina se corta en cortes de 4 micras, que se tiñen con hematoxilina-eosina para el estudio macroscópico.

En el estudio macroscópico y microscópico siempre se investigan y se describen los siguientes aspectos en relación con el tumor y la pieza: 1) tipo histológico del tumor y clasificación TNM; 2) Gleason; 3) focalidad tumoral, identificando el nódulo mayor; 4) localización del tumor y extensión a estructuras vecinas; 5) relación con la cápsula, grasa periprostática, vesículas seminales, plexos neurovasculares, eyaculadores, cuello vesical y ápex; 6) relación del tumor con los márgenes de resección, describiendo la forma de contacto (romo/espiculado) en caso de contacto, y tamaño aproximado; 7) presencia de PIN y su localización en relación con el tumor; 8) lesiones del resto de la próstata como hiperplasia, prostatitis, metaplasias y cambios postraumáticos.

De las 103 piezas de prostatectomía analizadas $5(4,9 \%)$ se clasificaron como pT0, $28(27,2 \%)$ pT2a, 26 (25,2\%) pT2b, 8 (7,8\%) pT2c, 25 (24,2\%) pT3a y $11(10,7 \%)$ pT3b. El Gleason se estableció en $5(4,8 \%)$ pacientes Gleason 3-4, 66 (64\%) pacientes Gleason 5-6 y 32 (31\%) pacientes en Gleason 7-10.
Biopsia preoperatorio y expresión de Ki67. Las biopsias preoperatorias se tomaron por punción con aguja 18 Gauge, guiadas por ecografia transrectal. Sistemáticamente se extraían como mínimo 6 muestras aleatorias, en dos planos parasagitales, derecho e izquierdo, 2 en ápex, 2 en zona media y 2 en zona basal. También se biopsiaban las áreas sospechosas, cuando existían, por lo que el número mínimo de biopsias analizadas siempre fue de 6. El material de cada zona biopsiada se fijaba en formol y se remitía por separado para estudio anatomopatológico. Se analizaban histológicamente de forma sistemática todos los cilindros de la biopsia. El número medio de cilindros biopsiados en cada paciente fue de 12,6 (rango 6-32, mediana 12).

En los 103 pacientes estudiados se determinó retrospectivamente el Ki67 en el material de las biopsias ecodirigidas preoperatorias. La población total del estudio fue de 103 casos que fueron obtenidos a partir de un listado de 252 pacientes intervenidos de prostatectomía radical entre julio de 1992 y diciembre de 2002. Las muestras histológicas utilizadas para el estudio fueron cilindros prostáticos embebidos en parafina, cuyos diámetros oscilan entre 0,2 y menos de $0,1 \mathrm{~cm}$. De cada uno de los casos fue elegido el cilindro con mayor volumen de parénquima ocupado por tumor, según los datos del informe anatomopatológico. En una primera selección, fueron rechazadas las muestras con material insuficiente, aquellas no conservadas en condiciones óptimas, las que presentaban focos tumorales menores del $2 \%$ del volumen total del cilindro o con menos de 100 células neoplásicas.

La inmunotinción se realizó en el laboratorio del Servicio de Anatomía Patológica, utilizándose un equipo automatizado Benchmark de la firma Ventana Medical Systems (EE.UU.), distribuido en España por Atom. El desenmascaramiento antigénico empleó un tampón comercial basado en TRIS suministrado por el mismo proveedor Cell Conditioning Solution (CC1), referencia 950124 , Ventana - con una temperatura de trabajo de $100^{\circ}$ durante 30 minutos. La incubación de los anticuerpos fue de 30 minutos. En la inmunotinción propiamente dicha se utilizó el método de la estreptavidina-biotina-peroxidasa, con diaminobencidina como cromógeno. El anticuerpo usado fue el Ki67 de la casa Master Diagnóstica clon MIB1, referencia 211611 , dilución 1:50. 
Una vez realizada la técnica, se llevó a cabo una segunda selección, excluyéndose los cilindros cuya inmunotinción no fue claramente nuclear. Siempre que el volumen tumoral lo permitió, el contaje se hizo en áreas neoplásicas con grado histológico similar al componente predominante del grado de Gleason que había determinado previamente el patólogo. El contaje se hizo con microscopio óptico, sobre un total de 100 células como mínimo.

Controles post-operatorios. En todos los pacientes se establecieron controles periódicos con determinación de PSA en sangre a las 6 semanas de la intervención y posteriormente cada 6 meses de forma indefinida. En caso de PSA superior a $0,20 \mathrm{ng} / \mathrm{ml}$ en al menos dos determinaciones se diagnosticaba al paciente de recidiva bioquimica o fracaso bioquímico.

Análisis estadistico: Para el análisis estadístico empleamos el programa informático, SPSS versión 11.0 para Windows, de Microsoft. Las variables analizadas fueron factores pronósticos clásicos, el PSA preoperatorio, el Gleason, el estadio anatomopatológico, recidiva bioquímica y el Ki67, relacionado con la dinámica celular. Se hizo un análisis estadístico descriptivo y un estudio estadístico analítico, con regresión logística multivariante. Se utilizó como variable dependiente la recidiva bioquímica y como covariables $\mathrm{PSA}(>10 /$ $\leq 10)$, Ki67(>3\%/ $\leq 3 \%)$, Gleason $(\geq 7 /<7)$, estadio anatomopatológico (pT3/pT0-2). Se estimó la probabilidad de recidiva bioquímica y tiempo hasta la recidiva por el método de Kaplan-Meier. Comparamos variables con la prueba $\mathrm{t} d \mathrm{de}$ Student y el test de Chi-cuadrado. Los resultados de las curvas de Kaplan-Meier se comparan con el test de Mantel-Haenszel.

\section{RESULTADOS}

Variables. De los 103 pacientes evaluados por la expresión de Ki67 a los que se le había practicado prostatectomía radical, en 71 pacientes no se detectó recidiva bioquímica, lo que representa un porcentaje del 69\% y 32 (31\%) recidivaron (Tabla 1).

El PSA medio previo a la intervención era de $10,07 \mathrm{ng} / \mathrm{ml}$ en los pacientes que no recidivaron
Tabla 1

\begin{tabular}{lcc}
\hline & Frecuencia & Porcentaje \\
\hline No recidiva bioquímica & 71 & $69 \%$ \\
Sí recidiva bioquímica & 32 & $31 \%$ \\
\hline
\end{tabular}

Evolución de los 103 pacientes evaluados con Ki67 a los que se les había practicado prostatectomía radical. En 71 (69\%) no se detectó recidiva bioquímica y $32(31 \%)$ recidivaron.

frente a 20,90 en los que se detectó recidiva bioquímica( $p=0,0001)$. La suma media de los Gleason de las piezas de prostatectomía radical era de 6,03 en los pacientes que no recidivaron frente a 6,75 de los pacientes con recidiva bioquímica( $p=0,0001)$. La cifra media de células con expresión de Ki67 era de 3,9\% en los pacientes que no recidivaron frente a 5,05\% en los pacientes con recidiva bioquímica $(\mathrm{p}=0,030)$. Los tumores pT0-2 no recidivaron en $55(82,1 \%)$ y se detectó recidiva bioquímica en 12 (17,9\%) de los pacientes. Los tumores pT3 no recidivaron en 16 $(44,4 \%)$ de los pacientes y se observó recidiva bioquímica en 20 (55,6\%) de los pacientes (Tabla 2).

Análisis multivariante. En el análisis multivariante, el porcentaje de células con expresión de Ki67 en la biopsia preoperatoria, superior o inferior al 3\%, no es una variable con la posibilidad de predecir de una forma significativa la recidiva bioquímica del cáncer de próstata después de la prostatectomía radical. El coeficiente $\beta$ es de $-0,745$ sin significación estadística $(p=0,204$ (Tabla 3).

Las variables que más influyen en la recidiva bioquímica del cáncer de próstata después de la

Tabla 2

\begin{tabular}{lccc}
\hline Variable & $\begin{array}{c}\text { No recidiva } \\
\text { bioquimica } \\
\text { (valores medios) }\end{array}$ & $\begin{array}{c}\text { Si recidiva } \\
\text { bioquimica } \\
\text { (valores medios) }\end{array}$ & $\mathbf{p}$ \\
\hline PSA & $10,07 \mathrm{ng} / \mathrm{ml}$ & $20,90 \mathrm{ng} / \mathrm{ml}$ & 0,0001 \\
Gleason & 6,03 & 6,75 & 0,0001 \\
Ki67 & $3,95 \%$ & $5,05 \%$ & 0,030 \\
pT0-2 & $55(82,1 \%)$ & $12(17,9 \%)$ & 0,0001 \\
pT3 & $16(44,4 \%)$ & $20(55,6 \%)$ & \\
\hline
\end{tabular}

Se encuentran diferencias significativas entre los pacientes con recidiva bioquímica y los que no recidivaron, en cuanto a la tasa de PSA preoperatorio, Gleason, porcentaje de expresión de Ki67 y estadio anatomopatológico. 
Tabla 3

\begin{tabular}{lccc}
\hline Variable & Coeficiente $\beta$ & $\begin{array}{c}\text { Error } \\
\text { estándar }\end{array}$ & p \\
\hline Constante & 2,904 & 0,614 & 0,0001 \\
Ki67 $(>3 \% / \leq 3 \%)$ & $-0,745$ & 0,586 & 0,204 \\
PSA $(>10 / \leq 10)$ & $-1,349$ & 0,521 & 0,010 \\
Gleason $(\geq 7 /<7)$ & $-1,146$ & 0,547 & 0,036 \\
pT(pT3/pT0-2) & $-1,284$ & 0,537 & 0,017 \\
\hline
\end{tabular}

En el análisis multivariante, el porcentaje de células con expresión de Ki67, superior o inferior al 3\%, no es una variable que prediga de una forma significativa la recidiva biológica del cáncer de próstata después de la prostatectomía radical $(\mathrm{p}=0,204)$.

prostatectomía radical son el Gleason, el PSA y el Estadio Anatomopatológico de la pieza de prostatectomía. La posibilidad de recidiva bioquímica es del $83 \%$ en las biopsias en las que el Ki67 > 3\% frente al 70,6\% de las biopsias con Ki67 $\leq 3 \%$ (p=N.S), cuando permanecen constantes las otras variables: Gleason $\geq 7$, PSA $>10 \mathrm{y}$ Estadio pT3. La posibilidad de recidiva bioquímica es del 10,4\% en las biopsias en las que el Ki67 > 3\% frente al 5,2\% de las biopsias con Ki67 $\leq 3 \%$ (p=N.S), cuando permanecen constantes las otras variables: Gleason $<7$, PSA $\leq 10$ y Estadio pTO-2 (Tabla 4).

Tabla 4

Porcentaje de expresión de Ki67 $\begin{gathered}\text { Frecuencia de } \\ \text { recidiva } \\ \text { bioquimica }\end{gathered}$

Gleason $\geq 7$, PSA $>10$, Estadio $p T 3$

Ki67 >3\%

$83,5 \%$

Ki67 $\leq 3 \%$

$70,6 \%$

N.S.

Gleason $<7$, PSA $\leq 10$, Estadio pTO-2

Ki67 >3\%

$10,4 \%$

$\mathrm{Ki} 67 \leq 3 \%$

$5,2 \%$

Tiempo hasta la recidiva. Los resultados de las curvas de Kaplan-Meier indican que no hay diferencias significativas, en cuanto al tiempo hasta la recidiva, entre el grupo de pacientes con expresión de Ki67 > 3\% y el grupo de pacientes con Ki67 $\leq 3 \%$. El porcentaje pacientes libres de recidiva estimados a los 120 meses para el grupo con Ki67 >3\% - independientemente del Gleason, PSA y Estadio pT- es del 54\% y en el grupo de pacientes con $K i 67 \leq 3 \%$ es del $67 \%$, sin diferencias significativas entre los dos grupos (Test de MantelHaenszel, p=N.S.) (Fig. 1).

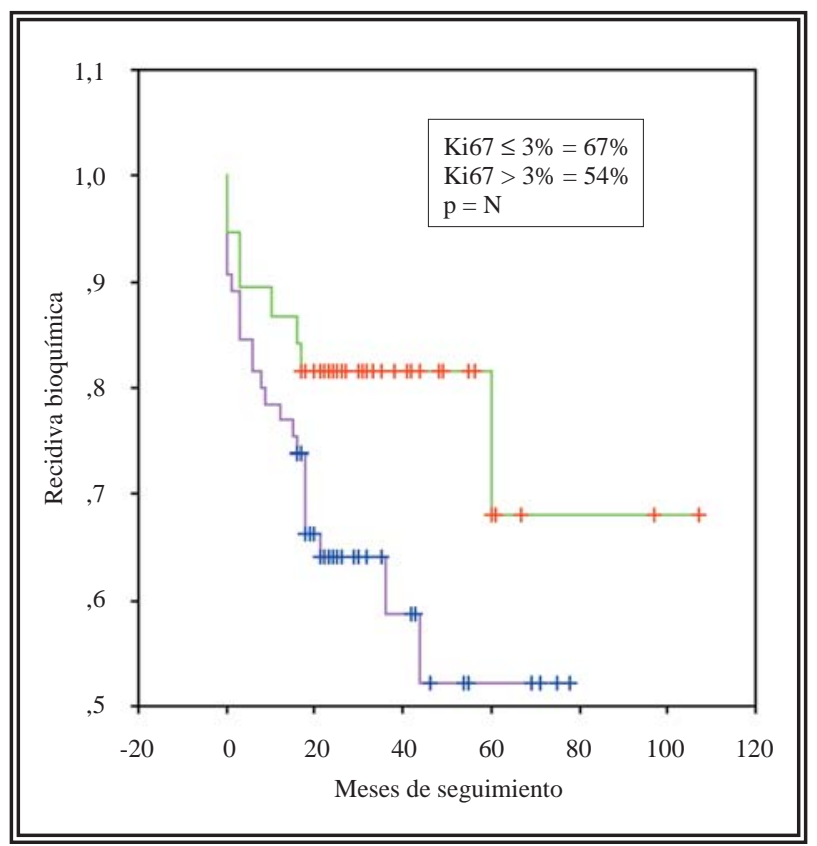

FIGURA 1. Los resultados de las curvas de Kaplan-Meier indican que no hay diferencias significativas, en cuanto al tiempo hasta la recidiva, entre el grupo de pacientes con expresión de Ki67 >3\% y el grupo de pacientes con Ki67 $\leq 3 \%$. La posibilidad de estar libre de recidiva a los 120 meses es del $67 \%$ en el grupo Ki67 $\leq 3 \%$ vs. $54 \%$ en el grupo Ki67 >3\% ( $p=N S)$.

Las variables que más influyen en la recidiva biológica son el Gleason, PSA y Estadio Anatomopatológico. La posibilidad de recidiva biológica es del $83 \%$ en las biopsias en las que el Ki67 $>3 \%$ frente al $70,6 \%$ de las biopsias con Ki67 $\leq 3 \%$ (p=N.S.), cuando permanecen constantes las otras variables: Gleason $\geq 7$, PSA $>10$ y Estadio pT3. La posibilidad de recidiva biológica es del 10,4\% en las biopsias en las que el Ki67>3\% frente al 5,2\% de las biopsias con Ki67 $\leq 3 \%$ ( $\mathrm{p}=\mathrm{N} . \mathrm{S})$, cuando permanecen constantes las otras variables: Gleason $<7$, PSA $\leq 10$ y Estadio pT0-2.

\section{COMENTARIOS}

En el cáncer de próstata los factores pronósticos que están relacionados con la biología molecular y la inmunohistoquímica se pueden clasificar como se expresa en la Tabla 5. Están vinculados con la estructura celular, la dinámica de la célula y sus ciclos, con la muerte celular por apoptosis y con la interfase o relación de la célula con el estroma que la rodea $6-12,14-51$. 
Los factores pronósticos relacionados con la estructura celular se asocian a proteínas estructurales y a la ploidía. Las proteínas estructurales, como la Catepsina D, EGFR, Proteína P, cerb2 y $p S 2$, se encuentran presentes en las membranas de las células. La sobre-expresión de la Catepsina D, EGFR, Proteína P, cerb2, sería indicativo de mal pronóstico y la sobre-expresión de pS2 de buen pronóstico. La ploidía es la cantidad de ADN de las células tumorales en relación con las células normales. Poliploidía y aneuploidía son sinónimo de más malignidad (Tabla 5).

Los factores pronósticos relacionados con el ciclo celular son proteínas asociadas a los nucleolos como la AgNORs, el antígeno nuclear proteico Ki67 y las Ciclinas que actúan a través de las Kinasas en las fases del ciclo celular. La sobreexpresión o porcentaje de células positivas augurarían mal pronóstico (Tabla 5).

Los factores pronósticos relacionados con la apoptosis o muerte celular son proteínas que actúan en el ciclo de la célula favoreciendo o retrasando la muerte de la célula. La proteína p27 bloquea el ciclo en G1 por la vía de las Ciclinas, la sobre-expresión indica buen pronóstico. La proteína p53 bloquea el ciclo en G1 y activa el fax y bax; contrariamente a lo esperado la sobre-expresión es un signo de mal pronóstico debido a que sólo se ve el p53 mutado que es el p53 que no es eficaz. La proteína BcL2 es una proteína mitocondrial que bloquea a la célula en fase GO e impide la muerte celular por apoptosis, inhibiendo el bax; la sobre-expresión indica mal pronóstico (Tabla 5).

Los factores pronósticos relacionados con la interfase célula estroma se clasifican por su mecanismo de acción en moléculas de adhesión y otros factores que intervienen en la neoangiogénesis y en las micrometástasis. Las moléculas de adhesión más importantes son la Cadherina $E$, relacionada con la adhesividad de las células tumorales, antimetástasis y buen pronóstico; la

\section{Tabla 5}

Algunos factores pronósticos del cáncer de próstata relacionados con la dinámica celular y el entorno que rodea a la célula.

\begin{tabular}{|c|c|c|c|}
\hline $\begin{array}{l}\text { Relacionados con la } \\
\text { estructura celular }\end{array}$ & $\begin{array}{l}\text { Relacionados con el } \\
\text { ciclo celular }\end{array}$ & $\begin{array}{l}\text { Relacionados con la } \\
\text { apoptosis }\end{array}$ & $\begin{array}{l}\text { Relacionados con la } \\
\text { interfase célula- } \\
\text { estroma }\end{array}$ \\
\hline Proteinas & AgNORs: Proteínas & Proteina p27: Inhibe & Moléculas de \\
\hline estructurales que & asociadas a los & el ciclo célula en G1 & adhesión: Cadherina \\
\hline están presentes en las & nucleolos. La & por la vía de las & $\boldsymbol{E}$, relacionada con la \\
\hline membranas. & sobreexpresión es de & Ciclinas. & adhesividad de las \\
\hline Sobreexpresión de & mal pronóstico & Sobreexpresión = a & células tumorales y \\
\hline mal pronóstico: & Ki67: Antígeno & buen pronóstico. & antimetástasis. \\
\hline Catepsina D, EGFR, & nuclear proteico, a & Proteina p53: Bloquea & Sobreexpresión = \\
\hline Proteina $P$, cerb2 & mayor porcentaje de & el ciclo en G1 y activa & buen pronóstico. \\
\hline Sobreexpresión de & células positivas & bax y fas. & Tenascina, sintetizada \\
\hline buen pronóstico: $\boldsymbol{p} \mathbf{S 2}$ & peor pronóstico. & Sobreexpresión = a & por las células que \\
\hline Ploidia & Ciclinas: Son & mal pronóstico porque & rodean al tumor. \\
\hline Que es la cantidad de & necesarias en todas & sólo se ve el p53 & Disminuye la \\
\hline ADN de las células & las fases del ciclo & mutado que no es & permeabilidad del \\
\hline tumorales, en & celular a través de & eficaz & estroma. \\
\hline relación con las & las Kinasas. La & Proteina BcL2: & Sobreexpresión = \\
\hline células normales & sobreexpresión es de & Proteína mitocondrial & mal pronóstico. \\
\hline Poliploidia y & mal pronóstico & que bloquea la célula & Metaloproteinas \\
\hline aneuploidía $=$ más & & en fase GO y la muerte & $(\mathrm{mm} 23, \mathrm{sLEX})$ \\
\hline malignidad & & por apoptosis, & proteinolíticas. \\
\hline & & inhibiendo el $\boldsymbol{b a x}$. & Sobreexpresión = \\
\hline & & Sobreexpresión = & mal pronóstico. \\
\hline & & mal pronóstico. & Neoangiogénesis \\
\hline & & & Micrometástasis \\
\hline
\end{tabular}


Tenascina, sintetizada por las células que rodean al tumor, que disminuye la permeabilidad del estroma y que es un dato de mal pronóstico; las metaloproteinas mm23 y sLEX son substancias proteinolíticas segregadas por el tumor, cuya sobre-expresión es un dato de mal pronóstico (Tabla 5).

El Ki67 por lo tanto es una proteína asociada al ciclo celular. En el cáncer de próstata el Ki67 ha sido empleado para diferenciar entre tejido prostático benigno y maligno, para investigar y confirmar factores pronósticos, como complemento del estudio histopatológico, para evaluar la respuesta al tratamiento y como factor pronóstico.

Ki67 y tejido prostático benigno y maligno. Rudolph P et al. en $1993^{14}$ describieron la diferencia de expresión de Ki67 entre el tejido prostático benigno y maligno. Los autores observaron que la expresión de Ki67 se relacionaba con el tipo de patología de la glándula prostática. El Ki67 se expresaba en el 0,35\% de las hiperplasias prostáticas benignas, la expresión oscilaba entre el 0,5 y $15 \%$ en los adenocarcinomas bien diferenciados, oscilaba entre el 1 y 40\% en los adenocarcinomas moderadamente diferenciados y alcanzaba al 80\% en los adenocarcinomas indiferenciados. Limas C et al. en $1994^{15}$ incorporaron la expresión de Ki67 para analizar la actividad proliferativa del epitelio prostático benigno y maligno. La expresión estaba aumentada en la inflamación, metaplasma y en el cáncer. En el cáncer la expresión de Ki67 no era homogénea pero estaba relacionada fundamentalmente con el grado. Helpap B en $1995^{16}$ investigó con técnicas de inmunohistoquímica y biología molecular la relación de la cinética celular con distintas patologías prostáticas, hiperplasia benigna de próstata, hiperplasia atípica adenomatosa, PIN y adenocarcinoma. Todas las técnicas demostraron una baja actividad en la hiperplasia benigna y en la hiperplasia atípica adenomatosa. La máxima actividad se manifestó en los adenocarcinomas de alto grado. En el PIN se situó entre los adenocarcinomas de grado intermedio. VoelkelJohnson C et al. en $2000^{17}$ desarrollaron animales de experimentación transgénicos con el objetivo de que se expresaran los dos oncogenes
ECO-RI y c-fos implicados en el cáncer de próstata. Observaron la aparición en las próstatas de los animales áreas de displasia, neoplasia intraepitelial y adenocarcinoma. El Ki67 estaba elevado y se relacionaba con la severidad de las lesiones prostáticas. Uzgare AR et al. en $2003^{18}$ analizaron el papel de tres proteínas de la familia de las Kinasas - erk, jnk y p38MAPK-en la iniciación y progresión del cáncer de próstata. Las conclusiones del estudio fueron que esas proteínas estaban ligadas a la iniciación del cáncer de próstata y a la expresión de Ki67 pero que no estaban asociadas a la evolución del cáncer de próstata ni a la diseminación metastásica. Fedosenko KV et al. en $2003^{19}$ analizan la expresión de Ki67, Bc12, p53 y ploidía en áreas peritumorales del cáncer de próstata y en zonas distantes correspondientes a hiperplasia prostática benigna. En el área peritumoral encontraron un alto porcentaje de células que expresaban el Ki67 y una baja inhibición de la apoptosis.

Ki67 para investigar y comparar factores pronósticos. Vartanian RK et al. en $1995^{20}$ analizaron con el Ki67 y otros métodos de estudio de la cinética celular la proliferación de las células endoteliales en el cáncer de próstata y en la hiperplasia benigna, relacionándola con el Gleason, la proliferación vascular y la proliferación de las células epiteliales. La proliferación endotelial en el cáncer de próstata es muy activa y es independiente de la proliferación vascular y de la actividad de las células epiteliales. Bubendorf L et al. en $1996^{21}$ estudiaron el significado pronóstico del Bcl-2 y Ki67 en el cáncer de próstata localizado. El análisis combinado de Ki67 y Bcl-2 permitía hacer 3 grupos diferentes de pacientes: tumores de buen pronóstico con Bcl-2 negativo y baja expresión de Ki67, tumores de mal pronóstico con $\mathrm{Bcl}-2$ positivo y alta expresión de Ki67 y tumores de pronóstico intermedio con combinaciones de Ki67 y Bcl-2. Aaltomaa S et al. en $1999^{22}$ evalúan la expresión de las ciclinas y su relación con diferentes variables clinicopatológicas y con la supervivencia. La expresión de la Ciclina D está relacionada con la expresión de Ki67. Lipponen P et al. en $2000^{23}$ relacionaron el Activador de la Proteína 2 con la diferenciación del cáncer de próstata y la proliferación celular. 
Observaron que una baja expresión del Activador de la Proteína 2 se relaciona con un alto índice mitótico y de expresión de Ki67. Giri D et al. en $2001^{24}$ demostraron con técnicas de inmunohistoquímica (Ki67) que la Interleukina-6 es importante factor de crecimiento autocrino en el cáncer de próstata hormonodependiente. Santamaría A et al. en $2003^{25}$ relacionaron los niveles altos de PTOV1 con un alto índice proliferativo que demostraban por una alta expresión del Ki67. Observaron una sobre-expresión de la proteína PTOV1 en el $71 \%$ de los cánceres de próstata y en el 80\% de las muestras con neoplasia intraepitelial. Pollack A et al. en $2003^{26}$ analizaron la relación entre la expresión de los biomarcadores Ki67, bcl-2, bax y bcl-x en pacientes con cáncer de próstata tratados con radioterapia externa. Observaron expresión de Ki67 > 3,5\% en el 33\% de los pacientes. Las anomalías en la expresión del bcl2 y del bax fueron asociadas a un incremento en el fracaso bioquímico de esos pacientes, por lo que podrían ser empleados para catalogar a ciertos tumores en grupos de alto riesgo de recidiva, independientemente de la expresión del Ki67 y de los factores de riesgo convencionales. Shim EH et al. en $2003^{27}$ observaron que la expresión de la proteína F-box SKP2 era inversamente proporcional a la expresión de la Ciclina p27 y Ki67 y que su sobre-expresión estaba ligada a áreas de hiperplasia, displasia o adenocarcinoma bien diferenciado en los animales de experimentación con cáncer de próstata.

\section{Ki67 como complemento del estudio histopatoló-} gico. Oxley JD et al. en $1998^{28}$ descubrieron por la expresión de Ki67 que los tumores ductales eran más indiferenciados que los tumores microacinares. Helpap B en $1998^{29}$ recomendó la determinación de diferentes métodos de análisis de la dinámica celular entre los que se encontraba el Ki67 para analizar el material de aquellas biopsias con un gradiente de Gleason bajo, para evitar infravaloraciones. Helpap B et al. en $1999^{30}$ investigaron la histogénesis de los adenocarcinomas indiferenciados de próstata analizando marcadores neuroendocrinos, receptores de andrógenos, PSA y la actividad proliferativa de las células con Ki67. Observaron 2 grupos de tumores. El primer grupo estaba formado por los tumores con marcadores neuroendocrinos negativos, PSA y receptores androgénicos positivos e índice de actividad mitótica del $34,8 \%$. El segundo grupo estaba formado por tumores con marcadores neuroendocrinos positivos, PSA y receptores androgénicos negativos y actividad mitótica del 82,6\%.

Ki67 para evaluar la respuesta al tratamiento. Szende B et al. en $2001^{31}$ estudiaron la evolución de 60 cánceres de próstata y concluyeron que el análisis de los índices de mitosis y apoptosis, expresión de Ki67 y p53 en repetidas biopsias permiten evaluar la eficacia del tratamiento $\mathrm{y}$ modificarlo según la conveniencia. Nicolai $\mathrm{H}$ et al. en $1994^{32}$ evaluaron con la expresión del Ki67 la respuesta de las células tumorales a los ultrasonidos. Revelaron que no existían cambios significativos en la distribución del ciclo celular con esa modalidad terapéutica. Montagnani Marelli M et al. en $1997^{33}$ analizaron los efectos de los agonistas LH-RH y la expresión del Ki67 en células tumorales de próstata in vivo e in vitro. La expresión de Ki67 era la misma in vivo que in vitro. Rittmaster RS et al. en $1999^{34}$ intentaron conocer el estado proliferativo y de apoptosis de la célula, para predecir el periodo hormonorrefractario del cáncer de próstata. La conclusión fue que la apoptosis desciende y la proliferación aumenta a medida que el cáncer se acerca a la fase hormonorrefractaria. Kelly WK et al. en $2000^{35}$ evaluaron los efectos de los retinoides en el cáncer de próstata. No observaron cambios histológicos ni en los índices de apoptosis y proliferativos en el 95\% de los casos, lo que sugería efectos antitumorales modestos. Van Leenders GJ et al. en $2000^{36}$ investigaron con la expresión de Ki67 los cambios histológicos en piezas de prostatectomía radical después de tratamiento con ultrasonidos de alta intensidad con Ki67. Según su estudio un gran volumen de glándula prostática quedaba sin tratamiento, con esa modalidad terapéutica. Szabo J et al. en $2000^{37}$ analizaron los efectos del tratamiento sobre el cáncer de próstata rebiopsiando a 10 pacientes que habían sido sometidos a bloqueo androgénico completo. La expresión del Ki67, bcl2 y p53 estaba altamente relacionada con tumores con Gleason superior a 7 y descendía de una forma importante en todos los casos, después del tratamiento. Romics I et al. en 
$2002^{38}$ concluyeron en el análisis de la evolución de 60 pacientes con cáncer de próstata que la expresión del Ki67 en las biopsias practicadas durante el tratamiento contribuía a evaluar la eficacia del tratamiento y a modificarlo. Polek TC et al. en $2003^{39}$ emplearon la determinación del Ki67 para evaluar la eficacia de la 1,25Dihydroxivitamina D en 2 líneas celulares de cáncer de próstata. La línea de células LNCaP fue mucho más sensible que la línea PC-3. En la línea de células tumorales LNCaP se observó una mayor cantidad de células bloqueadas en fase GO, menor expresión del Ki67 y una mayor inducción a la apoptosis. Oudard S et al. en $2003^{40}$ descubrieron, analizando la respuesta de líneas celulares tumorales prostáticas al Docetaxel y Mitoxantrone, que el Docetaxel presentaba un alto poder antitumoral, tanto en células hormonorresistentes como hormonosensibles. Curiosamente, los casos que respondían se asociaban a un incremento de células con expresión de Ki67, Her2/neu, baja expresión de CYP3A4 e inducción de múltiples anomalías mitóticas. Enmon R et al. en $2003^{41}$ investigaron el potencial de la $17-\mathrm{N}-$ allylamino-17-demethoxy geldanamycina (17AAG), un derivado de la geldanamycina, para sensibilizar las células tumorales de próstata a las radiaciones ionizantes. El efecto beneficioso del citostático se manifestó con la menor expresión de Ki67 en las preparaciones celulares. Szende B et al. en $2003^{42}$ estudiaron los efectos a corto plazo de los agonistas LH-RH en el cáncer de próstata, analizando los índices de apoptosis y de mitosis a las 24 horas, 7días y 30 días de la inyección del medicamento. Aunque observaron un notable descenso en los índices de proliferación, la expresión de Ki67 permaneció alta en todos los casos. Las células de 8 tumores sobre-expresaban el Ki67 entre un 2 y un 10\% y las células de 2 tumores lo sobre-expresaban en el 70 y $90 \%$.

Ki67 como factor pronóstico. Bubendorf L et al. en $1996^{43}$ analizaron las posibilidades del Ki67 para predecir la progresión del cáncer de próstata después de la prostatectomía radical. En su trabajo concluían que el Ki67 era un importante marcador pronóstico en el cáncer de próstata. Cher ML et al. en $1996^{44}$ observaron que los pacientes con metástasis ganglionares y con una fracción proliferativa del Ki67 menor de 0,1 vivían más que los pacientes con una fracción proliferativa de Ki67 mayor de 0,1, 8,7 años frente a 4,4 años. Bubendorf L et al. en $1998^{45}$ observaron en su análisis que el material de la biopsia sextante no sólo era útil para el diagnóstico del cáncer de próstata sino que también podía ser aprovechado para obtener información pronóstica, con el análisis de Ki67. La expresión media de células con Ki67 era de 7,5\% y tenía un alto poder predictivo para la supervivencia. Khoo VS et al. en $1999^{46}$ contrastaron la relación entre Ki67 y ploidía con la evolución del cáncer de próstata después de tratamiento con radioterapia externa. En los resultados distinguieron 3 categorías de tumores: Tumores con positividad de Ki67 $\leq$ al 1,5\%; tumores con positividad de Ki67 entre 1,5 y 3,5\%; tumores con positividad de Ki67 en un porcentaje mayor del 3,5\%. El Ki67 se comportó como un potente predictor de la evolución del paciente. Drobnjak M et al. en $2000^{47}$ asociaron la sobre-expresión de la Ciclina D con la presencia de metástasis óseas en el cáncer de próstata. En los casos estudiados la sobre-expresión de la Ciclina D también se asociaba a la sobreexpresión de Ki567. Cowen D et al. en $2002^{48}$ determinaron el significado del Ki67 como factor pronóstico del cáncer de próstata tratado con radioterapia. En un análisis de 106 pacientes el Ki67 se comportó como factor independiente con alto poder para predecir el fracaso bioquímico después del tratamiento con radioterapia. La capacidad para predecir la evolución del tumor no dependía del tipo de biopsia, independientemente de que hubiese sido tomada por resección transuretral o con aguja fina. Zellweger T et al. en $2003^{49}$ analizaron en el cáncer de próstata la expresión del Ki67, Bcl-2, p53, CD-10 y CD-138 por técnicas de inmunohistoquímica. El Ki67 y CD-138 eran predictores independientes de las recidivas tempranas y de peores supervivencias El Bcl-2 solamente predecía recidivas tempranas mientras que el p53 sólo peor supervivencia. La terapia hormonal neoadyuvante se asociaba con sobre-expresión de $\mathrm{Bcl}-2$, a inhibición de Ki67 y CD-10, y no afectaba a CD-138. Ben-Izhak O et al. en $2004^{50}$ vieron que la proteína Skp2 se asociaba con una alta tasa de células con expresión de Ki67 en las biopsias de pacientes con cánceres 
de próstata y concluyeron que podría ser utilizada como un marcador adicional para predecir la agresividad del tumor. En nuestro país Zudaire Bergera JJ et al. en $2000^{51}$ analizaron la relación del Ki67 de 67 piezas de prostatectomía con los datos clinicopatológicos y la evolución de los pacientes. La expresión media de Ki67 era de $7,2 \%$ (57\% casos $>5 \%$ ). Encontraron correlación entre la expresión de Ki67, p53, estadio clínico y recidiva bioquímica La expresión de Ki67 se relacionaba con un peor estadio, un alto porcentaje de recidiva bioquímica, con una influencia significativa en la supervivencia libre de recidiva en el análisis univariante, $76 \%$ vs $48 \%$ en 3 años.

En nuestro estudio, aunque la expresión de Ki67 es más elevada en las biopsias de los tumores con progresión bioquímica, en el análisis multivariante la expresión de Ki67 se mantuvo como una variable con menor capacidad para predecir la recidiva que el PSA, Gleason y Estadio Anatomopatológico. La posibilidad de recidiva bioquímica es del $83 \%$ para las biopsias en las que el $\mathrm{Ki} 67>3 \%$ y del 70,6\% en las biopsias con Ki67 $\leq 3 \%$ (p=N.S), cuando permanecen constantes Gleason >7, PSA $>10$ y Estadio pT3. La posibilidad de recidiva bioquímica es del 10,4\% para las biopsias en las que el Ki67 >3\% y del 5,2\% en las biopsias con $\mathrm{Ki} 67 \leq 3 \%$ ( $\mathrm{p}=\mathrm{N}$.S), con Gleason $<7$, PSA $\leq 10$ y Estadio pTO-2.

\section{CONCLUSIONES}

La expresión del Ki67 en las biopsias preoperatorias aporta poco a los factores pronósticos clásicos, PSA, Gleason o Estadio Anatomopatológico, para predecir la recidiva bioquímica del cáncer de próstata después de la prostatectomía radical.

\section{REFERENCIAS}

1. Partin AW, Yoo J, Carter HB, Pearson JD, Chan DW, Epstein JI, Walsh PC. The use of prostate specific antigen, clinical stage and Gleason score to predict pathological stage in men with localized prostate cancer. J Urol 1993 jul;150(1):110-114.

2. Wilkinson BA, Hamdy FC. State-of-the-art staging in prostate cancer. BJU Int 2001 mar;87(5):423-430.

3. Gilliland FD, Hoffman RM, Hamilton A, Albertsen P, Eley JW, Harlan L, Stanford JL, Hunt WC, Potosky A, Stephenson RA. Predicting extracapsular extension of prostate cancer in men treated with radical prostatectomy: results from the population based prostate cancer outcomes study. J Urol 1999 oct;162(4): 1341-1345.

4. Epstein JI, Potter SR. The pathological interpretation and significance of prostate needle biopsy findings: implications and current controversies. J Urol 2001 aug; 166(2):402-410.
5. Ojea Calvo A, Núñez López A, Dominguez Freire F, Alonso Rodrigo A, Rodríguez Iglesias B, Benavente Delgado J, Barros Rodríguez JM, Gómez-González MC, González Piñeiro A, Otero García M, Nogueira March JL. Correlation of the anatomopathological staging of radical prostatectomy specimens with the amount of cancer in the preoperative sextant biopsy. Actas Urol Esp 2003;27(6):428-437.

6. García Foucillas J, Bandrés E, Catalán V, García Amigot F, Zabalegui N. Conceptos básicos en biología molecular del cáncer. Susceptibilidad genética. ANALES Sis San Navarra 2000;24(Supl. 1):31-52.

7. Rudolph P, Lappe T, Kreipe H, Parwaresch MR, Schmidt D. Determination of proliferation activity of prostate cancers by means of nuclear proliferation-associated formalin resistant Ki-S5 antigens. Verh Dtsch Ges Pathol 1993;77:98-102.

8. Nicolai H, Steinbach P, Knuechel-Clarke R, Grimm D, Roessler W, Wieland WF, Hofstaedter F. Proliferation of tumor spheroids after shock-wave treatment. J Cancer Res Clin Oncol 1994; 120(7):438-441.

9. Limas C, Frizelle SP. Proliferative activity in benign and neoplastic prostatic epithelium. J Pathol 1994 nov;174(3):201-208.

10. Helpap B. Cell kinetic studies on prostatic intraepithelial neoplasia (PIN) and atypical adenomatous hyperplasia (AAH) of the prostate. Pathol Res Pract 1995 sep;191(9): 904-907.

11. Zellweger T, Ninck C, Mirlacher M, Annefeld M, Glass AG, Gasser TC, Mihatsch MJ, Gelmann EP, Bubendorf L. Tissue microarray analysis reveals prognostic significance of syndecan-1 expression in prostate cancer. Prostate 2003 apr 1;55(1):20-29.

12. Shim EH, Johnson L, Noh HL, Kim YJ, Sun H, Zeiss C, Zhang H. Expression of the F-box protein SKP2 induces hyperplasia, dysplasia, and low-grade carcinoma in the mouse prostate. Cancer Res 2003 apr 1;63(7):1583-1588.

13. Sobin LH, Wittekind CH: TNM Clasificación de los tumores malignos. Barcelona. Pulso Ediciones 2002:26-29.

14. Rudolph P, Lappe T, Kreipe H, Parwaresch MR, Schmidt D. Determination of proliferation activity of prostate cancers by means of nuclear proliferation-associated formalin resistant Ki-S5 antigens. Verh Dtsch Ges Pathol 1993;77: 98-102.

15. Limas C, Frizelle SP. Proliferative activity in benign and neoplastic prostatic epithelium. J Pathol 1994 nov;174(3): 201-208.

16. Helpap B. Cell kinetic studies on prostatic intraepithelial neoplasia (PIN) and atypical adenomatous hyperplasia (AAH) of the prostate. Pathol Res Pract 1995 sep;191(9): 904-907.

17. Voelkel-Johnson C, Voeks DJ, Greenberg NM, Barrios R, Maggouta F, Kurtz DT, Schwartz DA, Keller GM, Papenbrock T, Clawson GA, Norris JS. Genomic instability-based transgenic models of prostate cancer. Carcinogenesis 2000 aug; 21(8):1623-1627.

18. Uzgare AR, Kaplan PJ, Greenberg NM. Differential expression and/or activation of P38MAPK, erk1/2, and jnk during the initiation and progression of prostate cancer. Prostate 2003 may $1 ; 55(2): 128-139$

19. Fedosenko KV, Koval'skii GB. Proliferation and apoptosis of epithelium in prostate cancer, in a peritumor region, and in benign hyperplasia. Arkh Patol 2003 may-jun;65(3):18-20.

20. Vartanian RK, Weidner N. Endothelial cell proliferation in prostatic carcinoma and prostatic hyperplasia: correlation with Gleason's score, microvessel density, and epithelial cell proliferation. Lab Invest 1995 dec;73(6):844-850.

21. Bubendorf L, Sauter G, Moch H, Jordan P, Blochlinger A, Gasser TC, Mihatsch MJ. Prognostic significance of Bcl-2 in clinically localized prostate cancer. Am J Pathol 1996 may; 148(5): 1557-1565.

22. Aaltomaa S, Eskelinen M, Lipponen P. Expression of cyclin A and D proteins in prostate cancer and their relation to clinicopathological variables and patient survival. Prostate 1999 feb $15 ; 38(3): 175-182$. 
23. Lipponen P, Aaltomaa S, Kellokoski J, Ala-Opas M, Kosma V. Expression of activator protein 2 in prostate cancer is related to tumor differentiation and cell proliferation. Eur Urol 2000 may;37(5):573-578.

24. Giri D, Ozen M, Ittmann M. Interleukin-6 is an autocrine growth factor in human prostate cancer. Am J Pathol 2001 dec; 159(6):2159-2165.

25. Santamaría A, Fernández PL, Farre X, Benedit P, Reventos J, Morote J, Paciucci R, Thomson TM. PTOV-1, a novel protein overexpressed in prostate cancer, shuttles between the cytoplasm and the nucleus and promotes entry into the S phase of the cell division cycle. Am J Pathol 2003 mar;162(3):897905.

26. Pollack A, Cowen D, Troncoso P, Zagars GK, von Eschenbach AC, Meistrich ML, McDonnell T. Molecular markers of outcome after radiotherapy in patients with prostate carcinoma: Ki-67, bcl-2, bax, and bcl-x. Cancer 2003 apr 1;97(7):16301638.

27. Shim EH, Johnson L, Noh HL, Kim YJ, Sun H, Zeiss C, Zhang H. Expression of the F-box protein SKP2 induces hyperplasia, dysplasia, and low-grade carcinoma in the mouse prostate. Cancer Res 2003 apr 1;63(7):1583-1588.

28. Oxley JD, Abbott CD, Gillatt DA, MacIver AG. Ductal carcinomas of the prostate: a clinicopathological and immunohistochemical study. Br J Urol 1998 jan;81(1): 109-115.

29. Helpap B. Prognostic factors of prostatic carcinoma. Pathologe 1998 jan; 19(1):42-52.

30. Helpap B, Kollermann J. Undifferentiated carcinoma of the prostate with small cell features: immunohistochemical subtyping and reflections on histogenesis. Virchows Arch 1999 may;434(5):385-391.

31. Szende B, Romics I, Minik K, Szabo J, Torda I, Lovasz S, Szomor L, Toth L, Bely M, Kerenyi T, Bartok K, Vegh A Repeated biopsies in evaluation of therapeutic effects in prostate carcinoma. Prostate 2001 oct 1;49(2):93-100.

32. Nicolai H, Steinbach P, Knuechel-Clarke R, Grimm D, Roessler W, Wieland WF, Hofstaedter F. Proliferation of tumor spheroids after shock-wave treatment. J Cancer Res Clin Oncol 1994;120(7):438-441.

33. Montagnani Marelli M, Moretti RM, Dondi D, Limonta P, Motta M. Effects of LHRH agonists on the growth of human prostatic tumor cells: "in vitro" and "in vivo" studies. Arch Ital Urol Androl 1997 sep;69(4):257-263.

34. Rittmaster RS, Thomas LN, Wright AS, Murray SK, Carlson K, Douglas RC, Yung J, Messieh M, Bell D, Lazier CB. The utility of tissue transglutaminase as a marker of apoptosis during treatment and progression of prostate cancer. Urol 1999 dec;162(6):2165-2169.

35. Kelly WK, Osman I, Reuter VE, Curley T, Heston WD, Nanus DM, Scher HI. The development of biologic end points in patients treated with differentiation agents: an experience of retinoids in prostate cancer. Clin Cancer Res 2000 mar;6(3):838-846.

36. Van Leenders GJ, Beerlage HP, Ruijter ET, de la Rosette JJ, van de Kaa CA. Histopathological changes associated with high intensity focused ultrasound (HIFU) treatment for localised adenocarcinoma of the prostate. J Clin Pathol 2000 may;53(5):391-394.

37. Szabo J, Bartok K, Kereni T, Vegh A, Romics I, Szende B. Follow-up of prostate carcinoma patients treated with total androgen blockade by repeated map-biopsy. Ann Urol (Paris) 2000 aug;34(4):236-239.

38. Romics I, Lovasz S, Szabo J, Szomor L, Minik K, Bartok K, Kerenyi T, Szende B. The role of repeated biopsies in prognosis of prostate cancer. Orv Hetil 2002 jul 7;143(27): 16191623.

39. Polek TC, Stewart LV, Ryu EJ, Cohen MB, Allegretto EA Weigel NL. p53 Is required for 1,25-dihydroxyvitamin D3- induced G0 arrest but is not required for G1 accumulation or apoptosis of LNCaP prostate cancer cells. Endocrinology 2003 jan;144(1):50-60.

40. Oudard S, Legrier ME, Boye K, Bras-Goncalves R, de Pinieux G, De Cremoux P, Poupon MF. Activity of docetaxel with or without estramustine phosphate versus mitoxantrone in androgen dependent and independent human prostate cancer xenografts. J Urol 2003 may;169(5):1729-1734.

41. Enmon R, Yang WH, Ballangrud AM, Solit DB, Heller G, Rosen N, Scher HI, Sgouros G. Combination treatment with 17-Nallylamino-17-demethoxy geldanamycin and acute irradiation produces supra-additive growth suppression in human prostate carcinoma spheroids. Cancer Res 2003 dec 1;63(23):8393-8399.

42. Szende B, Lovasz S, Farid P, Romics I. Apoptosis in prostate carcinomas after short-term treatment with decapeptyl. Ann N Y Acad Sci 2003 dec;1010:784-788.

43. Bubendorf L, Sauter G, Moch H, Schmid HP, Gasser TC Jordan P, Mihatsch MJ. Ki67 labelling index: an independent predictor of progression in prostate cancer treated by radical prostatectomy. J Pathol 1996 apr; 178(4):437-441.

44. Cher ML, Stephenson RA, James BC, Carroll PR. Cellular proliferative fraction of metastatic lymph nodes predicts survival in stage D1 (TxN+M0) prostate cancer. J Urol 1996 may; 155(5): 1674-1677.

45. Bubendorf L, Tapia C, Gasser TC, Casella R, Grunder B, Moch H, Mihatsch MJ, Sauter G. Ki67 labeling index in core needle biopsies independently predicts tumor-specific survival in prostate cancer. Hum Pathol 1998 sep;29(9):949-954.

46. Khoo VS, Pollack A, Cowen D, Joon DL, Patel N, Terry NH, Zagars GK, von Eschenbach AC, Meistrich ML, Troncoso P. Relationship of Ki-67 labeling index to DNA-ploidy, S-phase fraction, and outcome in prostate cancer treated with radiotherapy. Prostate 1999 nov 1;41(3):166-172.

47. Drobnjak M, Osman I, Scher HI, Fazzari M, Cordón-Cardo C. Overexpression of cyclin D1 is associated with metastatic prostate cancer to bone. Clin Cancer Res 2000 may; 6(5): 1891-1895.

48. Cowen D, Troncoso P, Khoo VS, Zagars GK, von Eschenbach AC, Meistrich ML, Pollack A. Ki-67 staining is an independent correlate of biochemical failure in prostate cancer treated with radiotherapy. Clin Cancer Res 2002 may;8(5):1148-1154.

49. Zellweger T, Ninck C, Mirlacher M, Annefeld M, Glass AG, Gasser TC, Mihatsch MJ, Gelmann EP, Bubendorf L. Tissue microarray analysis reveals prognostic significance of syndecan-1 expression in prostate cancer. Prostate 2003 apr $1 ; 55(1): 20-29$.

50. Ben-Izhak O, Lahav-Baratz S, Meretyk S, Ben-Eliezer S, Sabo E, Dirnfeld M, Cohen S, Ciechanover A. Inverse relationship between Skp2 ubiquitin ligase and the cyclin dependent kinase inhibitor p27Kipl in prostate cancer. J Urol 2003 jul; $170(1): 241-245$.

51. Zudaire Bergera JJ, Sánchez Zalabardo D, Arocena GarcíaTapia J, Sanz Pérez G, Diez Caballero F, López Ferrandis J, Rosell Costa D, Robles García JE, de Álava E, Berián Polo JM. Expresión de p53 Ki67 en piezas de prostatectomía radical. Relación con los datos clínico-patológicos y supervivencia. Actas Urol Esp 2000 apr;24(4):307-313.

Dr. A. Ojea Calvo

Servicio de Urología. Hospital Univ. Xeral-Cíes de Vigo

C/ Pizarro, 22

36204 Vigo (Pontevedra)

(Trabajo recibido el 10 junio de 2004) 